\title{
BMJ Open Randomised comparative effectiveness trial of Pulmonary Embolism Prevention after hiP and kneE Replacement (PEPPER): the PEPPER trial protocol
}

\author{
Vincent D Pellegrini, Jr (D) , ${ }^{1}$ John William Eikelboom (D) , ${ }^{2} \mathrm{C}$ McCollister Evarts, ${ }^{3}$ \\ Patricia D Franklin (D) , ${ }^{4}$ Kevin L Garvin (D) , ${ }^{5}$ Samuel Z Goldhaber, ${ }^{6}$ Richard lorio, ${ }^{7}$ \\ Carol Ann Lambourne (D) , ${ }^{1}$ Jay Magaziner, ${ }^{8}$ Laurence Magder, ${ }^{8}$ Steering \\ Committee of the PEPPER Trial and the PEPPER Trial Investigators, funded by \\ PCORI
}

To cite: Pellegrini, Jr VD, Eikelboom JW, Evarts CMcC, et al. Randomised comparative effectiveness trial of Pulmonary Embolism Prevention after hiP and kneE Replacement (PEPPER): the PEPPER trial protocol. BMJ Open 2022;12:e060000. doi:10.1136/ bmjopen-2021-060000

- Prepublication history for this paper is available online. To view these files, please visit the journal online (http://dx.doi. org/10.1136/bmjopen-2021060000).

Received 08 December 2021 Accepted 01 February 2022

D) Check for updates

(c) Author(s) (or their employer(s)) 2022. Re-use permitted under CC BY-NC. No commercial re-use. See rights and permissions. Published by BMJ.

For numbered affiliations see end of article.

Correspondence to Dr Vincent D Pellegrini, Jr; vincent.d.pellegrini.jr@ dartmouth.edu

\section{ABSTRACT}

Introduction More than 1 million elective total hip and knee replacements are performed annually in the USA with $2 \%$ risk of clinical pulmonary embolism (PE), $0.1 \%-0.5 \%$ fatal $\mathrm{PE}$, and over 1000 deaths. Antithrombotic prophylaxis is standard of care but evidence is limited and conflicting. We will compare effectiveness of three commonly used chemoprophylaxis agents to prevent all-cause mortality (ACM) and clinical venous thromboembolism (VTE) while avoiding bleeding complications.

Methods and analysis Pulmonary Embolism Prevention after HiP and KneE Replacement is a large randomised pragmatic comparative effectiveness trial with non-inferiority design and target enrolment of 20000 patients comparing aspirin (81 mg two times a day), low-intensity warfarin (INR (International Normalized Ratio) target 1.7-2.2) and rivaroxaban $(10 \mathrm{mg} /$ day). The primary effectiveness outcome is aggregate of VTE and ACM, primary safety outcome is clinical bleeding complications, and patient-reported outcomes are determined at 1, 3 and 6 months. Primary data analysis is per protocol, as preferred for non-inferiority trials, with secondary analyses adherent to intention-to-treat principles. All non-fatal outcomes are captured from patient and clinical reports with independent blinded adjudication. Study design and oversight are by a multidisciplinary stakeholder team including a 10-patient advisory board. Ethics and dissemination The Institutional Review Board of the Medical University of South Carolina provides central regulatory oversight. Patients aged 21 or older undergoing primary or revision hip or knee replacement are block randomised by site and procedure; those on chronic anticoagulation are excluded. Recruitment commenced at 30 North American centres in December 2016. Enrolment currently exceeds 13500 patients, representing $33 \%$ of those eligible at participating sites, and is projected to conclude in July 2024; COVID-19 may force an extension. Results will inform antithrombotic choice by patients and other stakeholders for various risk cohorts, and will be disseminated through academic publications, meeting presentations and communications to advocacy groups and patient participants.
Strengths and limitations of this study

- Large pragmatic randomised comparative effectiveness trial of chemoprophylaxis in preventing venous thromboembolism (VTE) and all-cause mortality after elective total hip and total knee replacement.

- Target enrolment of 20000 patients provides power for concurrent analysis of both effectiveness (VTE) and safety (bleeding) endpoints.

- Non-inferiority design with primary per protocol analysis and secondary analysis by intention-totreat principles.

- A 10-patient advisory board contributed to the design and conduct of the trial, emphasising outcomes of importance to patients.

- Interim statistical analysis slated for $50 \%$ and $75 \%$ of target enrolment with 6-month follow-up and stopping criteria for significant differences in both VTE and bleeding.

Trial registration NCT02810704.

\section{INTRODUCTION}

\section{Background and significance}

Elective total joint arthroplasty (TJA) of the hip (THA) and knee (TKA) comprise the most commonly performed elective surgeries in the USA, totaling 1.2 million procedures annually. Hospital readmission within 90 days occurs after $10 \%-15 \%$ of elective THA and TKA, and venous thromboembolism (VTE), specifically pulmonary embolism (PE), can be lifethreatening. ${ }^{1-10}$ Despite advances in surgical care, fatal PE is reported in $0.1 \%-0.5 \%$ of patients and results in more than 1000 deaths annually following these operations. ${ }^{11-13}$ Postoperative anticoagulation to reduce thrombosis must be tempered by the bleeding risk implicit in orthopaedic procedures. 
Accordingly, orthopaedic surgeons typically opt for less intensive, while medical physicians favour more intensive, antithrombotics for TJA patients and clinical guidelines have historically been conflicted. Ideal thromboprophylaxis represents a balance between the risk of fatal PE and the treatment-associated morbidity of bleeding resulting in haematoma or secondary prosthetic infection. ${ }^{14}{ }^{15}$ Such a critical decision requires definitive evidence about potential benefits and harms, as well as consideration of individual patient preferences about these tradeoffs and risks.

Despite nearly five decades of TJA, there remain substantial evidence gaps concerning VTE prophylaxis. ${ }^{16-20}$ Guidelines from the American College of Chest Physicians (ACCP) and American Academy of Orthopaedic Surgeons (AAOS) have historically been at odds, resulting in confusion for both patient and physician. ${ }^{21-23}$ Reconciliation occurred in 2012 with the 2nd $\mathrm{AAOS}^{24}$ and 9th $\mathrm{ACCP}^{25}$ clinical guidelines wherein the ACCP provided greater emphasis on patient concerns about untoward bleeding. ${ }^{26}$ Both groups agreed that clinical VTE was the critical endpoint and acknowledged that insufficient data existed to provide endorsement of any preferred regimen. Few more compelling scenarios demand genuine consideration of outcomes important to patients than an elective operation with a reliable track record of improving quality of life that also carries a small, but real, risk of death and a greater risk of morbid complications that compromise function.

The Pulmonary Embolism Prevention after HiP and KneE Replacement (PEPPER) trial is a large pragmatic randomised clinical trial with non-inferiority design comparing three guideline-approved pharmacological agents popular in North America for prevention of VTE after THA and TKA. Clinical equipoise supports randomisation to three drugs that span the continuum of antithrombotic intensity. Aspirin, $81 \mathrm{mg}$ two times a day, represents low intensity and least costly treatment with minimal perceived bleeding risk and low rates of clinical PE/all-cause mortality (ACM) rates comparable to more intensive therapy. ${ }^{27}$ Rivaroxaban, $10 \mathrm{mg} /$ day, is a potent oral direct Factor Xa inhibitor, well-studied in randomised controlled trials, with very low VTE rates but higher bleeding risk $(3 \%-5 \%) .{ }^{28-33}$ Low intensity (INR 1.5-2) warfarin represents a compromise in anticoagulation intensity and has a delayed onset of action; it is historically one of the most commonly used agents having demonstrated effectiveness, low bleeding risk (1\%-2\%) and low cost. ${ }^{34}$ Each regimen is endorsed by clinical guidelines of the ACCP, AAOS and American College of Surgeons Surgical Care Improvement Project ${ }^{24} 2535$ (table 1).

The hypothesis is that aspirin prophylaxis of clinically meaningful VTE and death after THA and TKA will not be inferior to low intensity warfarin (INR 2.0) or rivaroxaban, and will result in fewer bleeding events with less reoperation, infection and myocardial infarction, that compromise patient-reported outcomes (PROs) for general well-being and joint-specific function.
Table 1 Clinical event risks for aggregate bleeding and pulmonary embolism associated with the three antithrombotics studied ${ }^{53}$

\begin{tabular}{lll}
\hline Study medication & $\begin{array}{l}\text { Risk of } \\
\text { reoperation for } \\
\text { bleeding }\end{array}$ & $\begin{array}{l}\text { Risk of } \\
\text { pulmonary } \\
\text { embolism }\end{array}$ \\
\hline Aspirin & 1 in $500(0.2 \%)$ & 1 in $50(2.0 \%)$ \\
Warfarin & 1 in $100(1.0 \%)$ & 1 in $100(1.0 \%)$ \\
Rivaroxaban & 1 in $20(5.0 \%)$ & 1 in $200(0.5 \%)$ \\
\hline
\end{tabular}

\section{METHODS AND ANALYSIS}

\section{Study setting and site selection}

The clinical coordinating centre (CCC) originated at the Medical University of South Carolina and transferred to Dartmouth-Hitchcock with relocation of the study PI (Principal Investigator). The data coordinating centre (DCC) resides at University of Maryland. An executive oversight committee (EOC) governs study operations. A patient advisory board ( $\mathrm{PAB})$, steering committee (SC), and data and safety monitoring board (DSMB) meet semi-annually (figure 1).

Enrolment occurs at North American THA and TKA referral centres (PEPPERstudy.org). Site requirements are: (1) willingness of individual participating surgeons to randomise eligible patients to all three treatment groups; (2) a minimum of two participating surgeons; (3) institutional experience with clinical trials; (4) existing research infrastructure or commitment to develop one; (5) adequate THA/TKA volume to provide enrolment of 1000 patients over 3 years; and (6) in the USA or Canada.

Primary reasons for site exclusion include: (1) unwillingness to randomise to all three groups; (2) budgeting or contractual limitations and (3) competing or conflicting studies. Institutions exhibiting structural barriers to use of warfarin (no INR monitoring) or rivaroxaban (no copay support) limit patient randomisation to only two treatment groups.

\section{Eligibility}

\section{Inclusion criteria}

Males and females 21 years of age or older undergoing unilateral elective hip or knee replacement, primary or revision, and medically eligible for randomisation to at least two of the study drugs.

\section{Exclusion criteria}

Patients undergoing bilateral THA or TKA; previously enrolled in PEPPER; concurrently enrolled in another active interventional VTE prophylaxis trial; or on chronic (longer than prior 6 months) anticoagulation. Patients with documented gastrointestinal, cerebral or other haemorrhage within 3 months; a diagnosis of defective hemostasis and history of spontaneous bleeding requiring transfusion; having an operation involving the eye, ear or central nervous system within 1 month; uncontrolled hypertension (systolic blood pressure (BP) $>220 \mathrm{~mm}$ $\mathrm{Hg}$ or diastolic $\mathrm{BP}>120 \mathrm{~mm} \mathrm{Hg}$ ); inadequate cognitive 


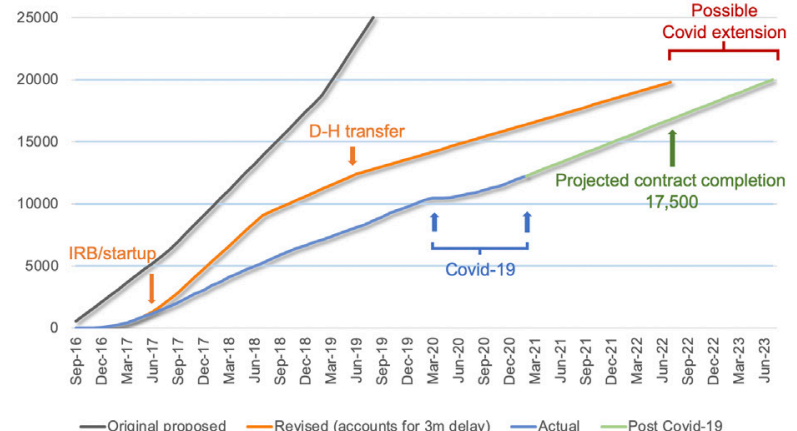

Figure 1 Pulmonary Embolism Prevention after HiP and KneE Replacement trial enrolment progression. Establishment of central IRB finalised 3 months after study contract signed with Patient Centered Outcomes Research Institute in March 2016. Study enrolment commenced in December 2016. Progressively more sites were added during first 12 months of trial; average time for contracting and infrastructure startup to enrolment ranged from 6 to 9 months. Study PI and prime contract transferred home institutions in June 2019. COVID-19 impact suspended elective surgery and research activity in nearly all sites commencing on or following March 2020 with sporadic resumption of research activities by 1 year later. Extension of the trial may be necessary as a result of the negative impact of COVID-19 on elective surgery and clinical research activities in the USA and Canada. IRB, institutional review board.

capacity to complete study assessments, or baseline body weight less than $41 \mathrm{~kg}(90.4 \mathrm{lbs})$. Pregnant or breastfeeding women of reproductive potential without a negative pregnancy test on day of surgery, and vulnerable populations including prisoners and institutionalised individuals are ineligible. Premenopausal women of childbearing potential include those not having had bilateral oophorectomy, hysterectomy or tubal ligation. Postmenopausal is defined as no menstrual period for one calendar year.

\section{Patient screening and consent}

Study subjects must meet protocol requirements, provide informed consent and agree to be randomised. Consent follows an introduction by the surgeon and an in-person discussion with a trained study team member. Live virtual remote consenting, implemented due to COVID-19 restrictions, and an educational video developed in conjunction with patients have been used.

All patients scheduled for elective THA or TKA are screened and all eligible patients are approached for participation. A patient is considered enrolled when all eligibility criteria are met and the institutional consent form (PEPPERstudy.org) is signed. Surgeon confirmation of eligibility is required prior to randomisation.

\section{Interventions}

Aspirin is initiated prior to surgery (162 mg po) on the day of operation. Starting postoperative day \#1, patients receive $81 \mathrm{mg}$ po two times a day.

Warfarin is initiated prior to surgery on the day of operation. Initial dosing is by body weight: less than $125 \mathrm{lbs}-2.5 \mathrm{mg}$; 125-250 lbs-5 mg; greater than 250 lbs-7.5 mg. The initial dose is repeated on the evening of surgery if the preoperative dose was received before noon. Starting postoperative day \#1, patients receive adjusted dose warfarin each evening to achieve a target INR of 2.0 (range 1.7-2.2). ${ }^{34} 36$ Monitoring is per local practice.

Rivaroxaban (10 mg po) is initiated on postoperative day \#1, 24 hours after arrival in the recovery room. Starting postoperative day \#2, rivaroxaban is administered each evening.

Study medications continue for 30 days in all groups. Continuation of study medication after any primary study endpoint or adverse event is subject to judgement of the treating physician. Data collection continues to complete 6-month follow-up. Patients on preoperative cardiac dose aspirin; (1) continue their regular cardiac dose if randomised to warfarin or rivaroxaban, or (2) change aspirin dosing to conform with PEPPER if randomised to the aspirin group. Pneumatic compression devices are used in all groups per local practice.

\section{Blinding}

Study group assignments are shared with the surgeon, clinical team and patient prior to operation. Follow-up data collection, event adjudications and statistical analyses are performed with blinding to treatment group.

\section{Randomisation, allocation, and implementation}

Randomisation occurs until the day before scheduled surgery and is available $24 / 7$ via a password protected time-stamped web-based system after baseline database instruments are completed. Random permuted blocks ensure balanced patient assignment to one of three treatment groups by operation and site. A contraindication to one of three regimens will result in 1:1 randomisation to the other two regimens. Following initial trial registration, the protocol was modified to allow limited select sites with structural impediments to use of either warfarin or rivaroxaban to omit that drug and randomise $1: 1$ to the other two drugs. Eligibility for two study regimens is required for patient participation in the trial.

Aspirin will be excluded from patients with:

1. Known aspirin allergy.

2. Personal history of PE/Deep Vein Thrombosis (DVT).

3. Proven thrombophilia by diagnostic testing, for example, Factor V Leiden.

4. Cancer diagnosis under active treatment.

Rivaroxaban will be excluded from patients:

1. With serum creatinine greater than $2.0 \mathrm{mg} / \mathrm{dL}$.

2. Taking medications that inhibit CYP 3A4.

Warfarin will be excluded from patients: 
1. With history of warfarin-related necrotising skin lesions.

\section{Patient and public involvement}

A multidisciplinary stakeholder team, including a 10-patient advisory board, participated in development of the research question and selection of both interventions and clinical outcomes to ensure consideration of patient priorities and preferences. Following initiation of the trial, the PAB advises on matters of patient recruitment, follow-up contact, adjunct studies, and will be involved in the analysis and interpretation of the final outcomes data. Results will be disseminated to all study participants who expressed interest in the outcomes through their local site of enrolment.

\section{Primary outcomes}

Clinical endpoints and functional outcomes are assessed through 6 months. The primary effectiveness endpoint is the aggregate of ACM and clinical VTE (PE, DVT), confirmed by imaging and resulting in readmission and/or therapeutic anticoagulation. Only the first or more serious event is counted in each patient. Deaths will be classified as cardiovascular, if sudden and otherwise unexplained, or myocardial infarction, stroke, heart failure, arrhythmia or PE. The primary safety endpoint is the aggregate of major bleeding, clinically important wound or remote bleeding, persistent wound drainage, reoperation for delayed wound healing or removal of the implant for infection, and myocardial infarction. Major bleeding is that which is fatal, occurs in a critical organ or space (intracranial, epidural, intraspinal, retroperitoneal, intraocular, pericardial), results in reoperation or remote clinically overt bleeding with a fall in haemoglobin of $20 \mathrm{~g} / \mathrm{L}$, managed with transfusion of two or more units of blood, or prolongs hospital stay. ${ }^{37}$ Non-major clinically important wound-related bleeding is persistent drainage beyond 5 days postoperatively or delayed healing that requires wound care after 2 weeks or staple removal. There is no VTE screening; clinical DVT is diagnosed by loss of compressibility on ultrasound ${ }^{39}$ or a filling defect on contrast venography. $\mathrm{PE}$ is diagnosed by contrast-enhanced chest $\mathrm{CT}^{40}{ }^{40}$ ventilation-perfusion scanning or pulmonary angiography. Myocardial infarction is diagnosed by laboratory-defined elevation in troponin and/or EKG changes. The primary functional endpoint is joint-specific PRO (hip (Hip Disability and Osteoarthritis Outcome Score (HOOS) $)^{41}$ or knee (Knee Disability and Osteoarthritis Outcome Score (KOOS) $)^{42}$ disability and osteoarthritis scores) and general well-being (PROMIS-10 global health ${ }^{43}$ at 1,3 and 6 months.

\section{Subgroup analyses}

Comparison of event rates in treatment subgroups defined by: (1) 'standard of care' anaesthesia methods (general vs regional/neuraxial $)^{2744} 45$ and (2) THA and TKA. Discordant patient $(\mathrm{PAB})$ and physician $(\mathrm{SC})$ anticoagulation preferences and risk tolerances will be reported.

\section{Sample size determination}

Sample size was designed to provide power to concurrently study both thrombosis and bleeding as surrogates for anticoagulant effectiveness and safety. Only clinically apparent events were considered. Conservative assumptions for fatal PE $(0.1 \%)$, clinical VTE $(2 \%)$ and clinical bleeding $(1 \%-5 \%)$ span the range of the three studied agents. Projections assumed enrolment of $22 \%$ of eligible patients reaching 25000 at 25 centres over 3 years. To date, enrolment of $34 \%$ of eligible patients has been achieved across all active sites.

Based on actual observed study event rates, the sample size was revised downward to 20000 participants. Conservatively assuming $10 \%$ of them will not be evaluable, we expect to have data on 18000 patients. Based on experience thus far, it is estimated that $7 \%$ of the patients will be ineligible for aspirin, $2.4 \%$ will be ineligible for rivaroxaban and $0.1 \%$ will be ineligible for warfarin. It is also estimated (based on the first 6 months of data and accounting for restricted randomisation at some sites) that $6.1 \%$ of those assigned to aspirin will not be treated with aspirin, $20 \%$ of those assigned to warfarin will not be treated with warfarin and $26.2 \%$ of those assigned to rivaroxaban will not be treated with rivaroxaban. These patients will be excluded from the primary 'per protocol' analysis after they switch medications. With a total sample of 18000 , considering the use of restricted randomisation at 10 sites and the $1-1$ randomisation, we project that for the per protocol analysis there will be 6050, 4306 and 4789 patients assigned to and treated in the aspirin, rivaroxaban and warfarin groups, respectively.

\section{Precision}

The primary endpoint is a composite consisting of the occurrence of death from any cause and clinically evident PE or DVT. We conservatively anticipate a primary endpoint in $2.5 \%$ of patients. For an observed endpoint that occurs $2.5 \%$ of the time, the two-sided $95 \%$ CI for the true proportion will be approximately $(2.03 \%$ to $2.97 \%$ ) for the rivaroxaban group and slightly narrower for the other two groups. Clinically significant bleeding is expected to occur in $4 \%-5 \%$ of patients on rivaroxaban, $1 \%-2 \%$ of patients on low-dose warfarin, and $0.5 \%-1 \%$ of patients on aspirin. For an observed proportion of $5 \%$, the two-sided $95 \%$ CI for the true proportion will be approximately $(4.33 \%$ to $5.67 \%)$; for an observed proportion of $0.5 \%$, the CI will be approximately $(0.28 \%$ to $0.72 \%)$. If the rate of events is equal to $2.5 \%$ in both the aspirin group and the rivaroxaban group, the expected $95 \%$ CI for the risk difference will be $(-0.65 \%$ to $+0.65 \%)$.

\section{Power}

The adoption of a non-inferiority methodology for this trial was predicated on establishing the non-inferiority of aspirin, as compared with either warfarin or rivaroxaban, in preventing death and thromboembolic disease with the assumption that aspirin would very likely result in fewer bleeding complications than either of these two 
agents. The statistical test of non-inferiority of aspirin relative to rivaroxaban will be based on only patients who were medically eligible for randomisation to either of those two groups. Allowing for $10 \%$ of patients with missing follow-up, accounting for those not treated with the study medication to which they were assigned, and excluding those who were at sites that did not randomise to rivaroxaban, we project that there will be 3942 patients in the rivaroxaban group and 4909 patients in the aspirin group. If the true risk of a primary endpoint is $2.5 \%$ for patients given either aspirin or rivaroxaban, the study will retain approximately $80 \%$ power to show that aspirin is not inferior to rivaroxaban by more than 0.94 percentage points, or a $37.6 \%$ increase in the risk of an event, using a standard large-sample one-sided test and a 0.025 significance level. Equivalently, the probability will be $80 \%$ that a two-sided $95 \%$ CI for the risk difference between aspirin minus the risk with rivaroxaban will have upper limit less than $0.94 \%$. For the comparison of aspirin and Warfarin, we project that we will have 4395 and 5058 comparable patients in the two groups and that we will have $83 \%$ power to reject inferiority of aspirin to warfarin by the same $0.94 \%$ margin. For the additional comparison of warfarin and rivaroxaban, which was not the basis for selecting the non-inferiority methodology, we project to have 3509 and 3798 patients in the two groups, respectively, providing $80 \%$ power to reject inferiority by a margin of 1.03 percentage points.

Using these assumptions, the margin of inferiority (table 2) that will be detectable given the different event rates from a final sample of 20000 randomised patients is as follows.

For example, in the riva/aspirin comparison, if the true event rate is $3.0 \%$ (in both groups), there will be $80 \%$ power to reject inferiority by a margin of 1.023 percentage points (or equivalently, by a factor of 1.341). Greater actual event rates provide a smaller relative margin of non-inferiority and a slightly larger absolute percentage point difference of non-inferiority.

\section{Data collection and participant follow-up}

All events are audited locally at hospital discharge, and collected centrally by a blinded independent third party data service (Statix, LLC; Salt Lake City, Utah, USA) at 4 weeks ( +10 days), 3 months $( \pm 10$ days $)$, and 6 months (+3 months/ -1 month) after operation (table 3$)$. Serial efforts are made to obtain follow-up by mail, email, telephone and web-enabled online surveys. Statix personnel interview patients about adverse events and collect PROs (HOOS, KOOS, PROMIS-10), available at PEPPERstudy.org. Patients without central 6-month follow-up are contacted by site coordinators to complete surveys. Medical records from all treatment facilities are collected by local PEPPER staff for endpoint or adverse events (identified by patient-report or ad hoc discovery), and patients lacking 6-month follow-up. All endpoints are adjudicated by two independent physicians (Outcomes Assessment Committee) blinded to study groups.

\section{Data management/monitoring}

Biweekly electronic enrolment and randomisation data are archived, edited and reported as blinded files by the DCC to the CCC and EOC. Statix follow-up data are downloaded quarterly. Deaths are monitored in real time. Batched adverse event triage and adjudication occurs semiannually on patients who have passed the 6-month window. A distinct unblinded DCC statistician analyses and prepares DSMB reports every 6 months. Site data collection and processes are audited annually by the study team.

No outcome or endpoint data are exchanged with clinical sites. Enrolment and follow-up figures are made available in a secure password protected section of the trial website.

\section{Statistical methods/data analysis plan (at PEPPERstudy.org)}

The trial's conceptual basis is to establish non-inferiority of aspirin, compared with warfarin or rivaroxaban, in preventing VTE and death. It is anticipated that aspirin would result in fewer bleeding complications than either of the other treatments. While the ITT principle is the basis for analysis of superiority trials, ITT is biased towards finding non-inferiority because protocol violations and cross-overs tend to diminish differences between groups. ${ }^{46} 47$ Therefore, the primary analysis of non-inferiority will be a per protocol analysis of patients receiving the medication to which they were randomised. If a patient switches treatments, $\mathrm{s} /$ he will be included in a per protocol analysis only up until the time the treatment changed. ${ }^{46}$ In this pragmatic trial the goal is to estimate event risks in a real world setting. This per protocol analysis is not a

Table 2 Pulmonary Embolism Prevention after HiP and KneE Replacement inferiority margins with observed event rates for both aspirin comparison

\begin{tabular}{llllll}
\hline & \multicolumn{2}{l}{ Rivaroxaban/aspirin comparison } & & \multicolumn{2}{l}{ Warfarin/aspirin comparison } \\
\cline { 2 - 3 } \cline { 5 - 6 } Event rate (\%) & Absolute difference (\%) & Relative difference & & Absolute difference (\%) & Relative difference \\
\hline 2.5 & 0.935 & 1.374 & 0.902 & 1.361 \\
3.0 & 1.023 & 1.341 & 0.984 & 1.328 \\
3.5 & 1.102 & 1.315 & 1.060 & 1.303 \\
\hline
\end{tabular}

*StatisicalAnalysisPlan,ThePEPPERTrial.atwww.PEPPERstudy.org 


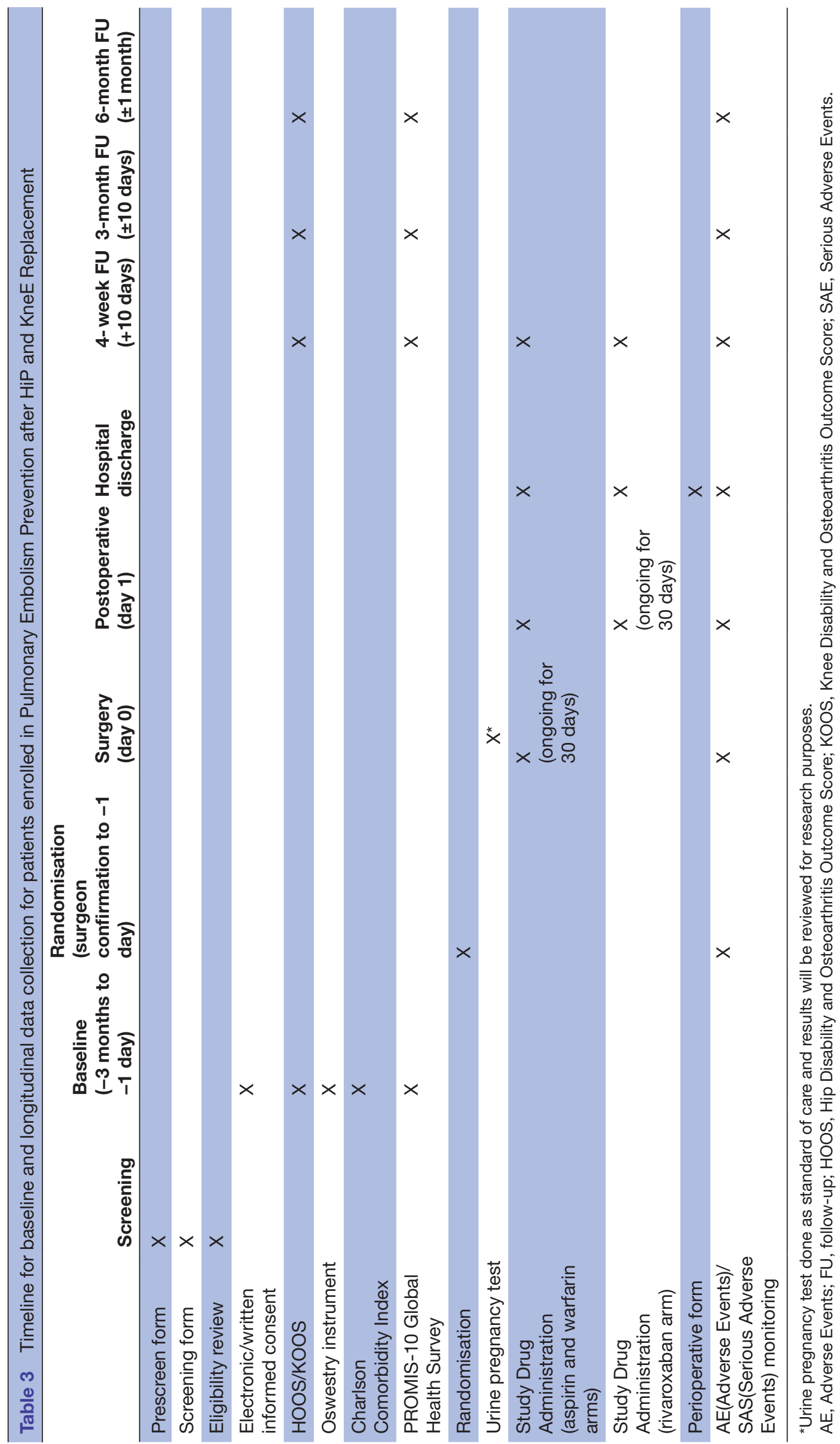

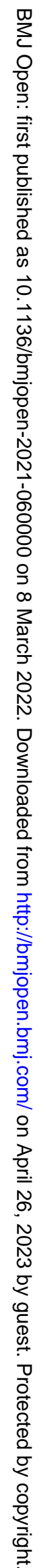


traditional one, which would exclude all patients who do not fully adhere to their medication assignment.

In secondary analyses, we will also perform intentionto-treat (ITT) analysis and 'as-treated' analyses. In the ITT analysis, patients will be included in the analysis in the group to which they were randomised and only be censored when they are lost to follow-up. In the 'as-treated' analysis, patients will be included in the group based on the study medication they actually received. This approach includes crossover patients and provides more precise estimates of what actually happened to patients who took the study medications, but will need to be interpreted with caution as it has more potential for a lack of group representativeness.

For each pairwise comparison of treatment groups, the statistical test of non-inferiority will be based on analysis of only patients eligible for randomisation to either of the two groups compared and only from sites when patients could have been randomised to either of the two treatments being compared. Defining the noninferiority margin is critical; it should be no more than the presumed entire effect of the active comparator. ${ }^{48}$ Projecting a primary endpoint (VTE plus ACM) in $2.5 \%$, a conservative non-inferiority margin for aspirin was defined as one percentage point or an effect no more than $40 \%$ greater than the comparator effect. With an actual primary endpoint in $1.5 \%$, the study will retain $80 \%$ power to show aspirin is not inferior to rivaroxaban by more than 0.73 percentage points $(49 \%)$ using a standard large-sample one-sided test $(\mathrm{p}=0.025)$. For comparison of aspirin and warfarin, $80 \%$ power is retained to reject inferiority by a similar margin $(0.71$ percentage points; 47\%). With 20000 patients, smaller actual event rates provide greater relative margins and slightly smaller absolute percentage point differences of non-inferiority (table 4).

A composite of primary effectiveness and safety endpoints will also be evaluated. This assessment of 'net clinical benefit' will compare a combined measure, the number of patients with either a primary effectiveness (DVT/PE plus ACM) or safety (aggregate bleeding events) endpoint between groups. ${ }^{49}$

\section{Interim analyses}

We do not anticipate stopping the trial early based on interim analyses of non-inferiority since evidence for inferiority with respect to both safety and efficacy should be present to end randomisation. The DSMB can recommend stopping randomisation to any medication (or modifying its dose) at any time based on safety concerns; prespecified outcomes leading to early stopping would be significant differences in (1) ACM or (2) both thrombotic and bleeding events. To preserve final $\mathrm{p}=0.05$ level type- 1 error rates for any specific comparison, the DSMB will use $p$ values of 0.002 at interim analyses of $50 \%$ and $75 \%$ of the sample to define statistical significance consistent with the Haybittle-Peto approach. ${ }^{50}$ The DSMB should consider stopping randomisation to a particular treatment if, at any interim analysis, (1) the two-sided p value comparing one treatment to another with respect to $A C M$ is lower than the corresponding threshold or (2) the twosided $\mathrm{p}$ values comparing one treatment to another with respect to both clinical thrombosis (DVT or PE) AND major bleeding events is lower than the corresponding threshold.

Table 4 Statistical power

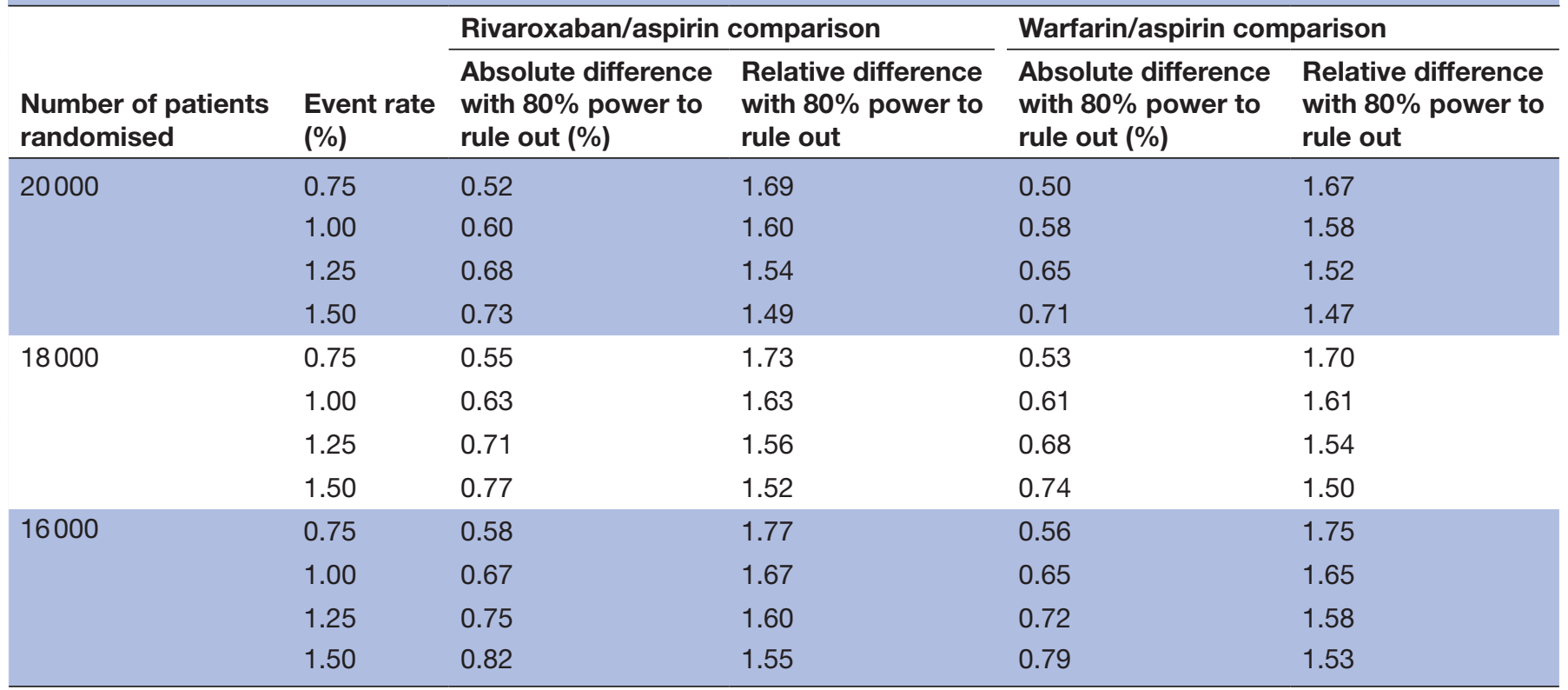

Absolute and relative margins of difference with $80 \%$ power to exclude with specified sample sizes and event rates. 


\section{ETHICS AND DISSEMINATION}

\section{Institutional Review Board approval}

The institutional review board (IRB) for human subjects research at the Medical University of South Carolina approved the study protocol and consent forms on 19 April 2016 (Pro00053742) and serves as the central IRB (cIRB). Of the 31 approved sites in North America, 27 relied on the cIRB; 2 USA and 2 Canadian sites are governed by local IRBs with guidance from the cIRB. Annual cIRB review was completed on 16 February 2021. Study documents and protocol modifications are available at PEPPERstudy.com. The study is funded by PCORI, and this report (Protocol version May 28, 2020) follows Standard Protocol Items: Recommendations for Interventional Trials guidelines.

\section{Safety monitoring}

An independent 5-member DSMB (two orthopaedic surgeons (one as chair), one haematologist, one cardiologist and one biostatistician) oversees study participant safety and overall trial conduct (charter at PEPPERstudy. org). Each member has signed, and regularly renews, a conflict of interest statement reporting relevant relationships. The DSMB reviews research protocol and informed consent documents; ensures data quality and study participant safety; reviews interim analyses; recommends early trial termination, modification or continuation; and advises the PI of any patient safety concerns or trial conduct recommendations. Study closure will be considered in consultation with the PI after outcome data and analyses are complete.

\section{Dissemination policy}

Publication of outcomes data will occur at project conclusion, consistent with normal scientific practices. Redacted data will be made available after main research findings are accepted for publication. Participating institutions will follow NIH data sharing guidelines. Ranges of adverse events related to VTE prophylaxis and associated recommendations will be provided using web-based applications consistent with policies of Dartmouth-Hitchcock Medical Center and the Trustees of Dartmouth College.

\section{Trial status}

As of 1 December 2021, 13663 patients have been enrolled, representing $33 \%$ of eligible patients at participating sites. With actual mortality of $0.3 \%$ and no more than 5\% loss to follow-up, a sample size of 20000 is projected by July 2024. Delays in central IRB implementation and the COVID-19 pandemic may extend enrolment beyond 6 years (figure 1). Experience finds ineligibility of $7 \%$ of patients for aspirin, $2.4 \%$ for rivaroxaban, and $0.1 \%$ for warfarin (figure 2 ).

\section{DISCUSSION}

PEPPER is the largest randomised trial of VTE prophylaxis after total joint replacement. It will provide much

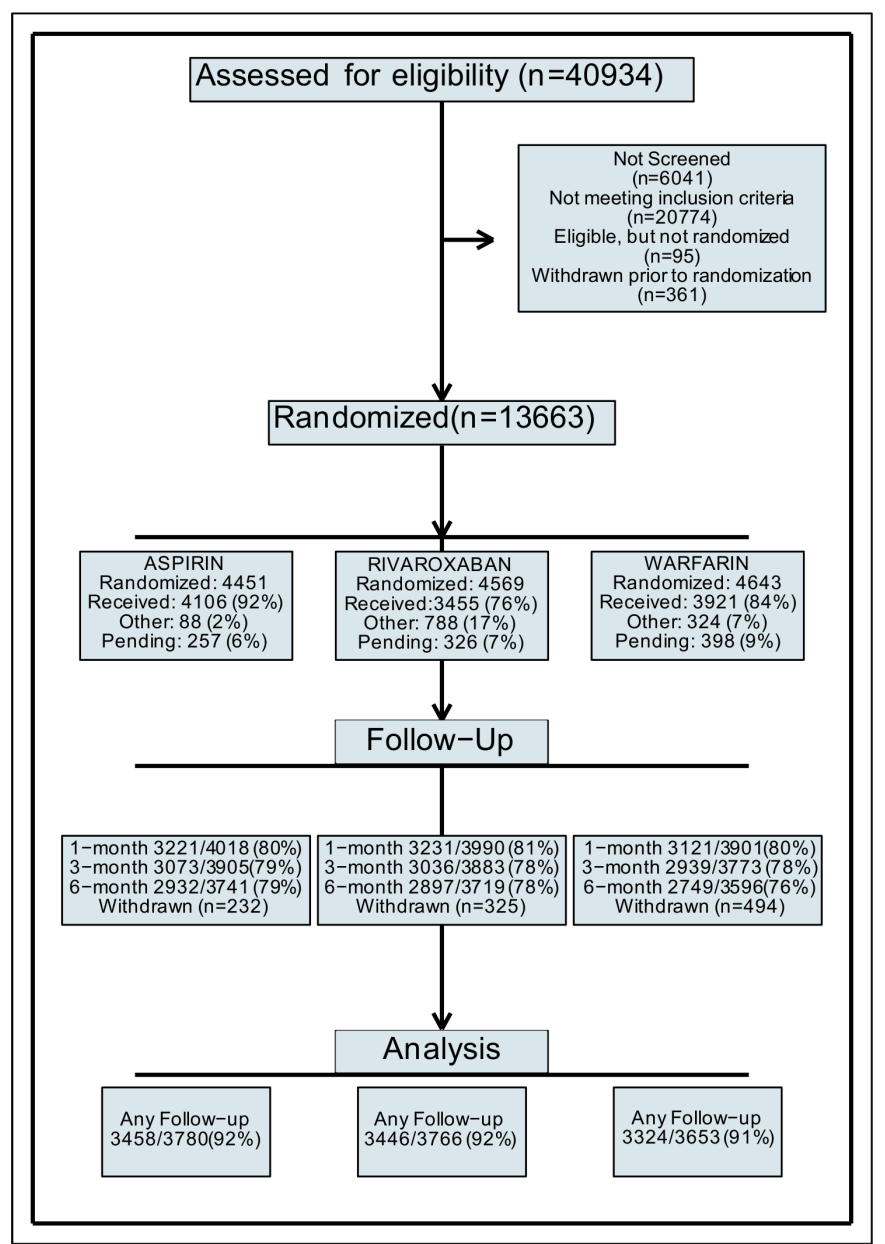

Figure 2 Pulmonary Embolism Prevention after HiP and KneE Replacement (PEPPER) trial Consolidated Standards of Reporting Trials (CONSORT) diagram. As of the end of November 2021, 13663 patients were randomised into PEPPER. More than $33 \%$ of all eligible patients undergoing total hip or knee replacement at participating centres agreed to participate in the trial. Approximately $80 \%$ of patients provided follow-up at 6 months through a centralised mechanism, as depicted in the CONSORT diagram. Local site efforts contribute to aggregate 6-month follow-up of greater than $95 \%$.

needed unbiased data regarding relative safety and efficacy of aspirin, rivaroxaban and warfarin and facilitate individualised prophylaxis for patients with various risk profiles. Similar actual event rates for clinically important effectiveness and safety endpoints will provide sufficient power for assessment of both thrombosis prevention and bleeding avoidance. As a large pragmatic trial, PEPPER reflects real world challenges related to drug cost, monitoring and adherence and their impact on outcomes. Since $25 \%$ of readmissions go to facilities other than that of the index procedure, patient event reporting will increase data on events of importance to patients, ${ }^{52}$ and a $\mathrm{PAB}$ ensures that endpoints and results interpretation will consider patient preferences. The PEPPER trial offers the prospect of objectively informing this critical patient care decision for more than 1 million individuals undergoing 
joint replacement annually in the USA at a time when rapid transition to expedited discharge and increasing financial constraints from bundled payments make the window of opportunity to conduct this important trial now or never.

\section{Author affiliations}

${ }^{1}$ Orthopaedics, Dartmouth-Hitchcock Medical Center, Lebanon, New Hampshire, USA

${ }^{2}$ Medicine, McMaster University Faculty of Health Sciences, Hamilton, Ontario, Canada

${ }^{3}$ Orthopaedics and Physical Medicine, Penn State College of Medicine, Hershey, Pennsylvania, USA

${ }^{4}$ Department of Medical Social Sciences, Northwestern University Feinberg School of Medicine, Chicago, Illinois, USA

${ }^{5}$ Orthopaedics and Physical Medicine, University of Nebraska Medical Center, Omaha, Nebraska, USA

${ }^{6}$ Medicine, Brigham and Women's Hospital, Boston, Massachusetts, USA ${ }^{7}$ Orthopaedics, Brigham and Women's Hospital, Boston, Massachusetts, USA ${ }^{8}$ Epidemiology and Public Health, University of Maryland School of Medicine, Baltimore, Maryland, USA

Collaborators The PEPPER Trial Investigators include James A. Browne, MD, University of Virginia; Eric M. Cohen, MD, Brown University/Lifespan Health; Charles M. Davis III, MD, PhD, Penn State University; Navin D. Fernando, MD, University of Washington; Kevin B. Fricka, MD, Anderson Orthopaedic Clinic; Richard J. Friedman, MD, Medical University of South Carolina; Kevin L. Garvin, MD, University of Nebraska; Richard lorio, MD, Brigham and Womens Hospital; Michael S. Kain, MD, Boston University; Stephen L. Kates, MD, Virginia Commonwealth University; Brent A. Lanting, MD, MSc, University of Western Ontario; Brock A. Lindsey, MD, West Virginia University; William J. Maloney, MD, Stanford University; Robert M. Molloy, MD, The Cleveland Clinic; Michael A. Mont, MD, Northwell Health; Wayne E. Moschetti, MD, MS, Dartmouth-Hitchcock Medical Center; James Nace, D0, MPT, Sinai Hospital, Baltimore; Charles L. Nelson, MD, University of Pennsylvania; Kevin I. Perry, MD, Mayo Clinic; James D. Slover, MD, New York University; Mark J. Spangehl, MD, Mayo Clinic Arizona; Lawrence M. Specht, MD, Lahey Clinic; Scott M. Sporer, MD, Rush University; Robert S. Sterling, MD, Johns Hopkins University; Zeke J. Walton, MD, Medical University of South Carolina. Data and Safety Monitoring Board: William L Healy, MD, Chair, Lahey Clinic and Medical Center; PJ Devereaux, MD, McMaster University; Charles W Francis, MD, University of Rochester; Patrick Heagerty, PhD, University of Washington; David J Warwick, DM MD BM FRCS (Ortho), University of Southampton. Executive Oversight Committee: Vincent D Pellegrini, Jr, MD, Chair and PI, Dartmouth-Hitchcock Medical Center and Geisel School of Medicine; Monica Baczko, MPA, Medical University of South Carolina; Patricia D Franklin, MD, MBA, MPH, Northwestern University; Carol A Lambourne, PhD, DartmouthHitchcock Medical Center; Jay S Magaziner, PhD, University of Maryland; Laurence S Magder, PhD, University of Maryland; Brook I. Martin, PhD, University of Utah. Outcomes Adjudication Committee: Samuel Z Goldhaber, MD, Chair, Brigham and Womens; Dale Adler, MD, Brigham and Womens Hospital; Rebecca Baron, MD, Brigham and Womens Hospital; Ronald Blankstein, MD, Brigham and Womens Hospital; Carolyn Come, MD, Lahey Clinic; Jean Connors, MD, Brigham and Womens Hospital; Raymond Kwong, MD, MPH, Brigham and Womens Hospital; Greg Piazza, MD, Brigham and Womens Hospital; Rachel Rosovsky, MD, Brigham and Womens Hospital; Aaron Waxman, MD, PhD, Brigham and Womens Hospital. Patient Advisory Board: Roger Bulger, MD, Co-Chair, Potomac, MD; C McCollister Evarts, MD, Co-Chair, Lancaster, PA; Ruth Bulger, PhD, Potomac, MD; Mary Beth Crummer, RN, Charleston, SC; Debra Hamilton, Charleston, SC; Nancy Mullen, Charleston, SC; Connie Persels, PhD, Potomac, MD; Robert Schlegel, PsyD, Charleston, SC; Sally Seeley, Cottonwood, AZ; Diane Smith, Charleston, SC; Mitchell Thompson, Charleston, SC (posthumously). Steering Committee: Teresa Bordeaux, National Blood Clot Alliance; John Eikelboom, MD, McMaster University; C McCollister Evarts, MD, Penn State Hershey Medical Center; Patricia D Franklin, MD, MBA, MPH, Northwestern University; Kevin L Garvin, MD, University of Nebraska; Samuel Z Goldhaber, MD, Harvard Medical School; Richard lorio, MD, Brigham and Womens Hospital; Carol A Lambourne, PhD, Dartmouth-Hitchcock Medical Center; Jay S Magaziner, PhD, University of Maryland; Laurence S Magder, $\mathrm{PhD}$, University of Maryland; Kathryn Z. Mikkelsen, North American Thrombosis Forum; Lisa K Moores, MD, Uniformed Services University of the Health Sciences.
Contributors Writing committee: VDP (PI and Chair), JWE, CME, PDF, KLG, SZG, $\mathrm{RI}, \mathrm{CAL}, \mathrm{JM}$ and $\mathrm{LM}$ all made substantial contributions to the manuscript. VDP, PDF, SZG, RI, CAL, JM, LM contributed to conception and design. VDP, JWE, CME, PDF, KLG, SZG, RI, CAL, JM, LM contributed to acquisition of data. VDP, JWE, CME, PDF, KLG, SZG, RI, CAL, JM, LM contributed to data analysis and interpretation. All authors read and approved the final manuscript.

Funding This work was supported by Patient Centered Outcomes Research Institute (PCORI), contract \# PCS-1402-09328-IC.

Competing interests VDP, JWE, CME, PDF, KLG, SZG, RI, CAL, JM and LM receive funding from PCORI in support of their work pursuant to this trial. JWE reports honoraria and grant support from Astra Zeneca, Bayer, Boehringer Ingelheim, Bristol-Myers-Squibb/Pfizer, Daiichi Sankyo, Glaxo Smith Kline, Janssen, Sanofi Aventis and Eli Lilly as well as a personal award from the Heart and Stroke Foundation. PDF reports grant support from PCORI, NIAMS, AHRQ and DePuy outside the submitted work. SZG reports grant support from Bayer, BoehringerIngelheim, BMS, Boston Scientific's BTG EKOS, Daiichi, Janssen, and NHLBI, and other from Bayer and Boehringer-Ingelheim outside the submitted work. JM reports grant support from the Patient Centered Outcomes Research Institute during the conduct of the study; personal fees from Novartis, Viking, Pluristem and UCB outside of the submitted work.

Patient and public involvement Patients and/or the public were involved in the design, or conduct, or reporting, or dissemination plans of this research. Refer to the Methods section for further details.

Patient consent for publication Not applicable.

Provenance and peer review Not commissioned; peer reviewed for ethical and funding approval prior to submission.

Open access This is an open access article distributed in accordance with the Creative Commons Attribution Non Commercial (CC BY-NC 4.0) license, which permits others to distribute, remix, adapt, build upon this work non-commercially, and license their derivative works on different terms, provided the original work is properly cited, appropriate credit is given, any changes made indicated, and the use is non-commercial. See: http://creativecommons.org/licenses/by-nc/4.0/.

\section{ORCID iDs}

Vincent D Pellegrini, Jr http://orcid.org/0000-0001-7492-0684

John William Eikelboom http://orcid.org/0000-0003-4126-1285

Patricia D Franklin http://orcid.org/0000-0002-4441-0533

Kevin L Garvin http://orcid.org/0000-0001-9288-8104

Carol Ann Lambourne http://orcid.org/0000-0003-4939-1746

\section{REFERENCES}

1 Charnley J. Low friction arthroplasty of the hip: theory and practice. Berlin: Springer-Verlag, 1979: 308.

2 Coventry MB, Beckenbaugh RD, Nolan DR, et al. 2,012 total hip arthroplasties. A study of postoperative course and early complications. J Bone Joint Surg Am 1974;56:273-84.

3 Coventry MB, Nolan DR, Beckenbaugh RD. "Delayed" prophylactic anticoagulation: a study of results and complications in 2,012 total hip arthroplasties. J Bone Joint Surg Am 1973;55:1487-92.

4 Insall JN. Surgery of the knee. New York: Churchill Livingstone, 1984. : 646-7p.

5 Johnson R, Green JR, Charnley J. Pulmonary embolism and its prophylaxis following the Charnley total hip replacement. Clin Orthop Relat Res 1977;127:123-32.

6 Murray DW, Carr AJ, Bulstrode CJ. Pharmacological thromboprophylaxis and total hip replacement. J Bone Joint Surg $\mathrm{Br}$ 1995;77:3-5.

7 Pellegrini VD, Donaldson CT, Farber DC, et al. The John Charnley Award: prevention of readmission for venous thromboembolic disease after total hip arthroplasty. Clin Orthop Relat Res 2005;441:56-62.

8 Pellegrini VD, Donaldson CT, Farber DC, et al. The mark coventry Award: prevention of readmission for venous thromboembolism after total knee arthroplasty. Clin Orthop Relat Res 2006;452:21-7.

9 Stulberg BN, Insall JN, Williams GW, et al. Deep-Vein thrombosis following total knee replacement. An analysis of six hundred and thirty-eight arthroplasties. J Bone Joint Surg Am 1984;66:194-201.

10 Yu S, Garvin KL, Healy WL, et al. Preventing Hospital readmissions and limiting the complications associated with total joint arthroplasty. J Am Acad Orthop Surg 2015;23:e60-71. 
11 Pellegrini VD. Prevention of thromboembolic disease in patients after total joint arthroplasty by selective post-hospitalization treatment. In: Fitzgerald RH, ed. Seminars in arthroplasty. Philadelphia: WB Saunders, 1997: 248-57.

12 Salvati EA, Pellegrini VD, Sharrock NE, et al. Recent advances in venous thromboembolic prophylaxis during and after total hip replacement. J Bone Joint Surg Am 2000;82:251-70.

13 Sculco TP, COLWELL CW, PELLEGRINI VD, et al. Prophylaxis against venous thromboembolic disease in patients having a total hip or knee arthroplasty. J Bone Joint Surg Am 2002;84:466-77.

14 Francis CW, Pellegrini VD, Harris CM, et al. Prophylaxis of venous thrombosis following total hip and total knee replacement using antithrombin III and heparin. Semin Hematol 1991;28:39-45.

15 Francis CW, Pellegrini VD, Marder VJ, et al. Prevention of venous thrombosis after total hip arthroplasty. antithrombin III and lowdose heparin compared with dextran 40. J Bone Joint Surg Am 1989;71:327-35.

16 US Department of Health and Human Services. NIH consensus development conference statement: prevention of venous thrombosis and pulmonary embolism. Bethesda, Maryland: US Department of Health and Human Services, 1986.

$17 \mathrm{NIH}$ Consensus Statement on Total Knee Replacement. NIH consensus and State-of-the-Science statements; us department of health and human services. Bethesda, Maryland, 2003.

18 Surgeon General's call to action to prevent deep vein thrombosis and pulmonary embolism. Available: http://www.surgeongeneral.gov/ topics/deepvein/calltoaction/call-to-action-on-dvt-2008.pdf

19 Agency for Healthcare Research and Quality. Effective health care program: venous thromboembolism prophylaxis in orthopedic surgery. Comparative effectiveness review number 49. AHRQ publication No. 12-EHC020-EF, 2012. Available: http:// effectivehealthcare.ahrq.gov/ehc/products/186/992/CER-49_VTE_ 20120313.pdf [Accessed 4 Apr 2013].

20 Pellegrini VD. Prophylaxis against venous thromboembolism after total hip and knee arthroplasty: a critical analysis review. JBJS Rev 2015;3:e1.

21 Eikelboom JW, Karthikeyan G, Fagel N, et al. American association of orthopedic surgeons and American College of chest physicians guidelines for venous thromboembolism prevention in hip and knee arthroplasty differ: what are the implications for clinicians and patients? Chest 2009;135:513-20.

22 Geerts WH, Bergqvist D, Pineo GF, et al. Prevention of venous thromboembolism: American College of chest physicians evidence-based clinical practice guidelines (8th edition). Chest 2008;133:381S-453.

23 Johanson NA, Lachiewicz PF, Lieberman JR. Prevention of symptomatic pulmonary embolism in patients undergoing total hip or knee arthroplasty. AAOS work group 20072009;17:183-96.

24 American Academy of Orthopaedic Surgeons. Preventing venous thromboembolic disease in patients undergoing elective hip and knee arthroplasty: evidence-based guideline and evidence report. Second edition. Rosemont, IL: AAOS, 2011. http://www.aaos.org/research/ guidelines/VTE/VTE full guideline.pdf

25 Falck-Ytter Y, Francis CW, Johanson NA, et al. Prevention of VTe in orthopedic surgery patients: antithrombotic therapy and prevention of thrombosis, 9th ED: American College of chest physicians evidencebased clinical practice guidelines. Chest 2012;141:e278S-325.

26 MacLean S, Mulla S, Akl EA, et al. Patient values and preferences in decision making for antithrombotic therapy: a systematic review: antithrombotic therapy and prevention of thrombosis, 9th ED: American College of chest physicians evidence-based clinical practice guidelines. Chest 2012;141:e1S-23.

27 Sharrock NE, Gonzalez Della Valle A, Go G, et al. Potent anticoagulants are associated with a higher all-cause mortality rate after hip and knee arthroplasty. Clin Orthop Relat Res 2008; $466: 714-21$.

28 Eriksson $\mathrm{BI}$, Borris LC, Friedman RJ, et al. Rivaroxaban versus enoxaparin for thromboprophylaxis after hip arthroplasty. $N$ Engl J Med 2008:358:2765-75.

29 Kakkar AK, Brenner B, Dahl OE, et al. Extended duration rivaroxaban versus short-term enoxaparin for the prevention of venous thromboembolism after total hip arthroplasty: a double-blind, randomised controlled trial. Lancet 2008;372:31-9.
30 Lassen MR, Ageno W, Borris LC, et al. Rivaroxaban versus enoxaparin for thromboprophylaxis after total knee arthroplasty. $N$ Engl J Med 2008;358:2776-86.

31 Turpie AGG, Lassen MR, Davidson BL, et al. Rivaroxaban versus enoxaparin for thromboprophylaxis after total knee arthroplasty (RECORD4): a randomised trial. Lancet 2009;373:1673-80.

32 Jensen CD, Steval A, Partington PF, et al. Return to theatre following total hip and knee replacement, before and after the introduction of rivaroxaban: a retrospective cohort study. J Bone Joint Surg Br 2011;93:91-5.

33 Jameson SS, Rymaszewska M, Hui ACW, et al. Wound complications following rivaroxaban administration: a multicenter comparison with low-molecular-weight heparins for thromboprophylaxis in lower limb arthroplasty. J Bone Joint Surg Am 2012;94:1554-8.

34 Lieberman JR, Wollaeger J, Dorey F, et al. The efficacy of prophylaxis with low-dose warfarin for prevention of pulmonary embolism following total hip arthroplasty. J Bone Joint Surg Am 1997;79:319-25.

35 Surgical care improvement project core measure set. Available: http://jointcommission.org/PerformanceMeasurement/PerformanceM easurement/SCIP+Core+Measure+Set.htm

36 Pellegrini VD. Warfarin prophylaxis for orthopaedic venous thromboembolic disease. instructional course lectures AAOS 51, 2002: 491-8.

37 Kucher N, Castellanos LR, Quiroz R, et al. Time trends in warfarinassociated hemorrhage. Am J Cardiol 2004;94:403-6.

38 Francis CW, Pellegrini VD, Totterman S, et al. Prevention of deep-vein thrombosis after total hip arthroplasty. Comparison of warfarin and dalteparin. J Bone Joint Surg Am 1997;79:1365-72.

39 Kearon C, Ginsberg JS, Hirsh J. The role of venous ultrasonography in the diagnosis of suspected deep venous thrombosis and pulmonary embolism. Ann Intern Med 1998;129:1044-9.

40 Stein PD, Fowler SE, Goodman LR, et al. Multidetector computed tomography for acute pulmonary embolism. N Engl J Med 2006;354:2317-27.

41 Nilsdotter AK, Lohmander LS, Klässbo M, et al. Hip disability and osteoarthritis outcome score (HOOS)--validity and responsiveness in total hip replacement. BMC Musculoskelet Disord 2003;4:10.

42 Roos EM, Lohmander LS. The knee injury and osteoarthritis outcome score (KOOS): from joint injury to osteoarthritis. Health Qual Life Outcomes 2003;1:64.

43 Hays RD, Bjorner JB, Revicki DA, et al. Development of physical and mental health summary scores from the patient-reported outcomes measurement information system (PROMIS) global items. Qual Life Res 2009;18:873-80.

44 Sharrock NE, Go G, Harpel PC. The John Charnley Award: thrombogenesis during total hip arthroplasty. Clin Orthop Relat Res 1995;319:16-27.

45 Dalldorf PG, Perkins FM, Totterman S, et al. Deep venous thrombosis following total hip arthroplasty. Effects of prolonged postoperative epidural anesthesia. J Arthroplasty 1994;9:611-6.

46 Hernán MA, Robins JM. Per-Protocol analyses of pragmatic trials. N Engl J Med 2017;377:1391-8.

47 Mauri L, D'Agostino RB. Challenges in the design and interpretation of Noninferiority trials. N Engl J Med 2017;377:1357-67.

48 Non-inferiority Clinical Trials to Establish Effectiveness: Guidance for Industry. US Department of Health and Human Services \& Food and Drug Administration, 2016. Available: https://www.fda.gov/media/ 78504/download [Accessed 31 Jul 2021].

49 Samama CM, Laporte S, Rosencher N, et al. Rivaroxaban or enoxaparin in Nonmajor orthopedic surgery. $N$ Engl J Med 2020;382:1916-25.

50 Haybittle JL. Repeated assessment of results in clinical trials of cancer treatment. Br J Radiol 1971:44:793-7.

51 Peto R, Pike MC, Armitage P, et al. Design and analysis of randomized clinical trials requiring prolonged observation of each patient. I. Introduction and design. Br J Cancer 1976;34:585-612.

52 Harrold L, Pascal S, Lewis C, et al. Patient report improves posthospital discharge event capture in total joint replacement: a novel approach to capturing all posthospital event data. EGEMS 2014;2:13.

53 Comparative effectiveness of pulmonary embolism prevention after hip and knee replacement (pepper): the pepper trial. Available: https://clinicaltrials.gov/ct2/show/NCT02810704 [Accessed 2 Aug 2021]. 